\title{
Species of Malva L. (Malvaceae) Cultivated in the Western of Santa Catarina State and Conformity With Species Marketed as Medicinal Plants in Southern Brazil
}

\author{
Leyza Paloschi de Oliveira ${ }^{1}$, Massimo Giuseppe Bovini ${ }^{2}$, Roseli Lopes da Costa Bortoluzzi ${ }^{1}$, \\ Mari Inês Carissimi Boff ${ }^{1} \&$ Pedro Boff ${ }^{3}$ \\ ${ }^{1}$ Programa de Pós-graduação em Produção Vegetal, Universidade do Estado de Santa Catarina, Lages, Santa \\ Catarina Brazil \\ ${ }^{2}$ Instituto de Pesquisas Jardim Botânico do Rio de Janeiro, Rio de Janeiro, Brazil \\ ${ }^{3}$ Laboratório de Homeopatia e Saúde Vegetal, Empresa de Pesquisa Agropecuária e Extensão Rural de Santa \\ Catarina (EPAGRI), Lages, Santa Catarina, Brazil \\ Correspondence: Leyza Paloschi de Oliveira, Programa de Pós-graduação em Produção Vegetal, Universidade \\ do Estado de Santa Catarina, Centro de Ciências Agroveterinárias, Av. Luiz de Camões 2090, Conta Dinheiro, \\ CEP 88.520-000, Lages, Brazil. Tel: 55-499-9912-6147. E-mail: leyza@uniarp.edu.br
}

Received: June 3, 2019

Accepted: July 12, 2019 Online Published: September 15, 2019

doi:10.5539/jas.v11n15p171

URL: https://doi.org/10.5539/jas.v11n15p171

\begin{abstract}
The Malva genus presents different species with therapeutic potential and inadequate consumption can occur due to the incorrect identification of the plant in the market. The objective of this study was to identify species of the Malva genus cultivated in the Western Mesoregion of Santa Catarina State-Southern Brazil, and to verify the conformity of products' labels marketed as dehydrated medicinal plants through the characteristics of the plant parts. The specimens were collected following an unsystematic procedure from households of ten municipalities. The material was identified with the help of specialized bibliography and a specialist. After, they were herborized and deposited in the herbaria of the State University of Santa Catarina (LUSC) and of the Botanical Garden Research Institute of Rio de Janeiro (RB). Five cultivated species of Malva were found (M. nicaeensis, M. parviflora, M. pseudolavatera, M. Sylvestris and M. verticillata). Whereas one species of the Geraniaceae family (Pelargonium graveolens) and three species of the Malvaceae family (Malva sylvestris, M. verticillata and Sida cordifolia) were found in the dehydrated packages. S. cordifolia species was present in $80 \%$ of the samples, with $65 \%$ of them marketed as M. sylvestris or under the common name of "mallow". Macroscopic images of the species and illustrations of the mericarps were made and an identification chart along with the morphological description for each species was elaborated based on the morphological results. Descriptions of labels for 60 samples were checked for the determination of the dehydrated Malva species marketed. Characteristics of parts of the plant in comparing them with the characteristics described in the specialized literature were performed. The target species of incorrect identifications in the analyzed packages was $M$. sylvestris.
\end{abstract}

Keywords: Malva sylvestris, medicinal plants, morphology, Sida cordifolia

\section{Introduction}

Medicinal plants represent an important therapeutic resource for the Brazilian population and they are part of the country's Public Policies (Brasil, 2006). The use of medicinal plants can be influenced by the economic condition, the high cost of medicines and the difficult access to public consultations. In addition to that, there is a difficulty of access by residents in rural areas to health care units located in urban areas. Moreover, the increase the trend for considering traditional knowledge that supports using natural resources as an alternative to synthetic drugs (Battisti et al., 2013).

The indiscriminate use of plants due to the lack of phytochemical, pharmacological and mainly toxicological knowledge is of great concern for public health. The correct identification of medicinal plant species is necessary, especially when they are processed in order to avoid misuse of medicinal plants (Romitelli \& Martins, 2013). The study of the plant's anatomy and morphology can help in the quality control of processed medicinal plants. 
The macroscopic and microscopic identifications of parts of the commercialized plants should be part of the current norms applied to drugs of plant origin (Brasil, 2010).

Malvaceae ('the mallows') is a botanical family with a rich diversity of species for textile, medicinal, and ornamental purposes. It consists of 4465 species and about 245 genera (The Plant List, 2013) and mallows present a cosmopolitan distribution, but with a high number of species in the tropics. In Brazil, there are 781 species with 73 genera distributed in all biomes (Flora do Brasil 2020, n.d.). Given its high diversity, its primary genetic center of origin is still discussed, and many species have become generalized over world (Ray, 1995). The Malva L. genus, popularly known as mallow, grows spontaneously in almost all of Europe and the Mediterranean region. The vast majority of the mallows genera are native to Brazil, with less than a dozen of which being considered as introduced species.

The Malva genus has 25-40 species and it can be considered as an annual and/or biannual herb. Flowers with an epicalyx and 8-15 reticulated mericarps are the typical one (Fryxell, 1988). Ray (1995) and Escobar, Schönswetter, Fuertes Aguilar, Neto Feliner and Schneeweiss (2009) relate their similarity to the Lavatera L. genus, where the bractoles of the epicalice are joined at the base, in contrast to in Malva where they are totally separated. Although there is inconsistency for some species in relation to the fusion of bracts and other characteristics. Molecular studies have also shown that the separation of these two genera based on this morphology is artificial and unsatisfactory (Escobar et al., 2009).

Malva species are indicated with potential therapeutic as cicatrizing and analgesic by the Ministry of Health. They are part of the National List of Medicinal Plants of interest of the SUS (Sistema Único de Saúde-RENISUS), whose objective is to guide the productive chain of medicinal plants and research development. Studies carried out by Alves (2009) and Benson (2015) consider M. sylvestris L. as having dental antimicrobial activities in oral treatments. According to the Phytotherapeutic Guidelines of Brazilian Pharmacopoeia, the indications for its use are the leaves and flowers for internal use such as an expectorant, and for external use as an antiseptic for the oral problems (Farmacopeia Brasileira, 2002). Ecker et al. (2015) compiled 56 articles addressing $M$. sylvestris as an herbal medicine for several purposes.

The use of M. sylvestris as a medicinal plant in Brazil involves different species, with the consumption of mallow leaves collected from plantations or acquired from commercial/health establishments for medicinal purposes. A survey of the conformity of macroscopic characteristics of parts of leaves, stems, floral buds and fruits of mallows marketed as medicinal plants, and the identification of the species grown near residences can clarify which species are commercialized.

The objectives of this study were to identify species of the genus Malva grown in the Western Mesoregion of the state of Santa Catarina, Southern Brazil, and to verify the conformity of the label of commercialized products, such as dehydrated medicinal plants, according to the characteristics of parts of the plant.

\section{Method}

Species were localized in households of ten municipalities in the Western Mesoregion of Santa Catarina State according to previous indication. The study was made among coordinates $26^{\circ} 35^{\prime} 58.91^{\prime \prime} \mathrm{S}$ to $27^{\circ} 22^{\prime} 22.97^{\prime \prime} \mathrm{S}$ and $50^{\circ} 40^{\prime} 17.00^{\prime \prime} \mathrm{W}$ to $52^{\circ} 36^{\prime} 36.79^{\prime \prime} \mathrm{W}$ at an altitude ranging from 450 to 1200 meters (Figure 1, Table 1). 


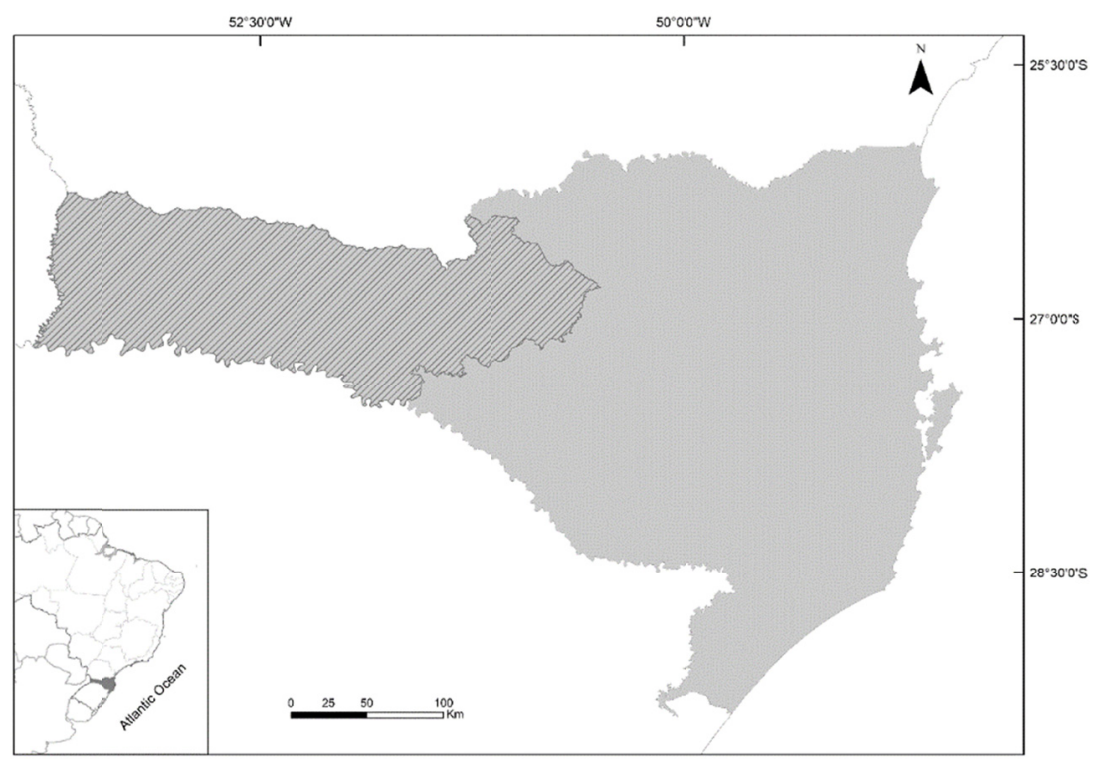

Figure 1. Map of the state of Santa Catarina (Brazil). Oblique lines delimit the Western Mesoregion of Santa Catarina

Malva species were collected in an unsystematic way, meaning without a defined system. The collected material was herborized in accordance with the usual herborization techniques and later incorporated into the Lages herbaria of the State University of Santa Catarina (LUSC) and the Botanical Garden Research Institute of Rio de Janeiro (RB). The morphological study was carried out based on the collected material, and the collections were deposited in the LUSC, RB and R herbaria (acronyms according to Thiers, 2017), as reference standards. Species were identified with the aid of specialized literature as described by Robyns (1965), Baroni (1984), Fryxell (1988), and Ray (1995). Consultation with specialists and often the support of digital platforms such as the Hinsley (2014) and Flora-On (2017) were used. ArcGis 10.5 software was used for preparing the map.

Table 1. Municipalities and coordinates of the sampled sites for Malva species grown in the western Mesoregion of Santa Catarina State, Brazil

\begin{tabular}{|c|c|c|c|c|c|c|c|}
\hline Municipality & Lat. (S) & Long. (W) & 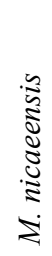 & 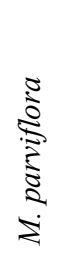 & 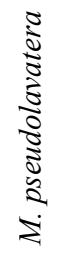 & 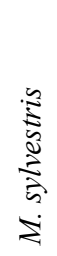 & 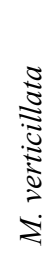 \\
\hline \multirow{7}{*}{ Caçador } & $26^{\circ} 46^{\prime} 44.7^{\prime \prime} \mathrm{S}$ & $51^{\circ} 0^{\prime} 47.1^{\prime \prime} \mathrm{W}$ & & $\mathrm{X}$ & & $\mathrm{X}$ & $\mathrm{X}$ \\
\hline & $26^{\circ} 44^{\prime} 13.6^{\prime \prime} \mathrm{S}$ & $50^{\circ} 59^{\prime} 56.9^{\prime \prime} \mathrm{W}$ & & $\mathrm{X}$ & & $\mathrm{X}$ & \\
\hline & $26^{\circ} 46^{\prime} 59.7^{\prime \prime} \mathrm{S}$ & $51^{\circ} 0^{\prime} 10^{\prime \prime} \mathrm{W}$ & & $\mathrm{X}$ & & & \\
\hline & $26^{\circ} 46^{\prime} 36.2^{\prime \prime} \mathrm{S}$ & $51^{\circ} 0^{\prime} 49.3^{\prime \prime} \mathrm{W}$ & & $\mathrm{X}$ & & & \\
\hline & $26^{\circ} 46^{\prime} 44.7^{\prime \prime} \mathrm{S}$ & $51^{\circ} 0^{\prime} 47.1^{\prime \prime} \mathrm{W}$ & & $\mathrm{X}$ & & & \\
\hline & $26^{\circ} 46^{\prime} 59.9^{\prime \prime} \mathrm{S}$ & $51^{\circ} 1^{\prime} 44.0^{\prime \prime} \mathrm{W}$ & & $\mathrm{X}$ & & & \\
\hline & $26^{\circ} 46^{\prime} 6.3^{\prime \prime} \mathrm{S}$ & $51^{\circ} 0^{\prime} 22.2^{\prime \prime} \mathrm{W}$ & & & & $\mathrm{X}$ & \\
\hline \multirow{9}{*}{ Calmon } & $26^{\circ} 35^{\prime} 58.91^{\prime \prime} \mathrm{S}$ & $51^{\circ} 6^{\prime} 2.52^{\prime \prime} \mathrm{W}$ & & & $\mathrm{X}$ & & $\mathrm{X}$ \\
\hline & $26^{\circ} 36^{\prime} 15.62^{\prime \prime} \mathrm{S}$ & $51^{\circ} 5^{\prime} 54.78^{\prime \prime} \mathrm{W}$ & & $\mathrm{X}$ & $\mathrm{X}$ & & \\
\hline & $26^{\circ} 36^{\prime} 14.61^{\prime \prime} \mathrm{S}$ & $51^{\circ} 5^{\prime} 54.92^{\prime \prime} \mathrm{W}$ & & $\mathrm{X}$ & & & \\
\hline & $26^{\circ} 36^{\prime} 15.47^{\prime \prime} \mathrm{S}$ & $51^{\circ} 5^{\prime} 54.82^{\prime \prime} \mathrm{W}$ & & $\mathrm{X}$ & & & \\
\hline & $26^{\circ} 36^{\prime} 15.3^{\prime \prime} \mathrm{S}$ & $51^{\circ} 5^{\prime} 54.8^{\prime \prime} \mathrm{W}$ & & $\mathrm{X}$ & & & \\
\hline & $26^{\circ} 36^{\prime} 12.4^{\prime \prime} \mathrm{S}$ & $51^{\circ} 5^{\prime} 59.1^{\prime \prime} \mathrm{W}$ & & $\mathrm{X}$ & & & $\mathrm{X}$ \\
\hline & $26^{\circ} 36^{\prime} 14.83^{\prime \prime} \mathrm{S}$ & $51^{\circ} 6{ }^{\prime} 2.80^{\prime \prime} \mathrm{W}$ & $\mathrm{X}$ & & & & \\
\hline & $26^{\circ} 36^{\prime} 14.83^{\prime \prime} \mathrm{S}$ & $51^{\circ} 6^{\prime} 2.16^{\prime \prime} \mathrm{W}$ & $\mathrm{X}$ & & & & \\
\hline & $26^{\circ} 36^{\prime} 13.93^{\prime \prime} \mathrm{S}$ & $51^{\circ} 5^{\prime} 44.08^{\prime \prime} \mathrm{W}$ & $\mathrm{X}$ & $X$ & & & \\
\hline
\end{tabular}




\begin{tabular}{|c|c|c|c|c|c|}
\hline Catanduvas & $27^{\circ} 4^{\prime} 13.98^{\prime \prime} \mathrm{S}$ & $51^{\circ} 39^{\prime} 42.01^{\prime \prime} \mathrm{W}$ & & & $\mathrm{X}$ \\
\hline Cordilheira Alta & $26^{\circ} 59^{\prime} 41.10^{\prime \prime} \mathrm{S}$ & $52^{\circ} 36^{\prime} 36.79^{\prime \prime} \mathrm{W}$ & $\mathrm{X}$ & $\mathrm{X}$ & \\
\hline \multirow{8}{*}{ Lebon Regis } & $26^{\circ} 59^{\prime} 59.53^{\prime \prime} \mathrm{S}$ & $50^{\circ} 43^{\prime} 57.14^{\prime \prime} \mathrm{W}$ & $\bar{X}$ & & \multirow{8}{*}{$X$} \\
\hline & $26^{\circ} 55^{\prime} 53.29^{\prime \prime} \mathrm{S}$ & $50^{\circ} 41^{\prime} 52.65^{\prime \prime} \mathrm{W}$ & $\mathrm{X}$ & & \\
\hline & $26^{\circ} 59^{\prime} 29.65^{\prime \prime} \mathrm{S}$ & $50^{\circ} 43^{\prime} 28.16^{\prime \prime} \mathrm{W}$ & $\mathrm{X}$ & & \\
\hline & $26^{\circ} 59^{\prime} 6.43^{\prime \prime} \mathrm{S}$ & $50^{\circ} 42^{\prime} 42.62^{\prime \prime} \mathrm{W}$ & $\mathrm{X}$ & & \\
\hline & $26^{\circ} 55^{\prime} 54.66^{\prime \prime} \mathrm{S}$ & $50^{\circ} 41^{\prime} 54.74^{\prime \prime} \mathrm{W}$ & $\mathrm{X}$ & & \\
\hline & $26^{\circ} 51^{\prime} 36.00^{\prime \prime} \mathrm{S}$ & $50^{\circ} 50^{\prime} 18.49^{\prime \prime} \mathrm{W}$ & $\mathrm{X}$ & & \\
\hline & $26^{\circ} 51^{\prime} 33.58^{\prime \prime} \mathrm{S}$ & $50^{\circ} 50^{\prime} 19.39^{\prime \prime} \mathrm{W}$ & $\mathrm{X}$ & & \\
\hline & $26^{\circ} 55^{\prime} 30.64^{\prime \prime} \mathrm{S}$ & $50^{\circ} 39^{\prime} 57.59^{\prime \prime} \mathrm{W}$ & $\mathrm{X}$ & & \\
\hline Peritiba & $27^{\circ} 22^{\prime} 22.97^{\prime \prime} \mathrm{S}$ & $51^{\circ} 54^{\prime} 14.00^{\prime \prime} \mathrm{W}$ & & & $\mathrm{X}$ \\
\hline \multirow{7}{*}{ Rio das Antas } & $26^{\circ} 54^{\prime} 9.93^{\prime \prime} \mathrm{S}$ & $51^{\circ} 4^{\prime} 35.65^{\prime \prime} \mathrm{W}$ & $\mathrm{X}$ & & \multirow{6}{*}{$X$} \\
\hline & $26^{\circ} 52^{\prime} 54.62^{\prime \prime} \mathrm{S}$ & $51^{\circ} 2^{\prime} 53.7^{\prime \prime} \mathrm{W}$ & $\mathrm{X}$ & & \\
\hline & $26^{\circ} 54^{\prime} 8.62^{\prime \prime} \mathrm{S}$ & $51^{\circ} 4^{\prime} 34.81^{\prime \prime} \mathrm{W}$ & & & \\
\hline & $26^{\circ} 52^{\prime} 52.31^{\prime \prime} \mathrm{S}$ & $51^{\circ} 7^{\prime} 35.56^{\prime \prime} \mathrm{W}$ & $\mathrm{X}$ & & \\
\hline & $26^{\circ} 53^{\prime} 29.07^{\prime \prime} \mathrm{S}$ & $51^{\circ} 3^{\prime} 36.755^{\prime \prime} \mathrm{W}$ & $\mathrm{X}$ & & \\
\hline & $26^{\circ} 53^{\prime} 39.22^{\prime \prime} \mathrm{S}$ & $51^{\circ} 4^{\prime} 28.88^{\prime \prime} \mathrm{W}$ & $\mathrm{X}$ & & \\
\hline & $26^{\circ} 53^{\prime} 54.59^{\prime \prime} \mathrm{S}$ & $51^{\circ} 4^{\prime} 30.86^{\prime \prime} \mathrm{W}$ & & & $\mathrm{X}$ \\
\hline \multirow{7}{*}{ Timbó Grande } & $26^{\circ} 37^{\prime} 7.60^{\prime \prime} \mathrm{S}$ & $50^{\circ} 40^{\prime} 26.79^{\prime \prime} \mathrm{W}$ & $\bar{X}$ & \multirow{7}{*}{$\mathrm{X}$} & \\
\hline & $26^{\circ} 37^{\prime} 9.94^{\prime \prime} \mathrm{S}$ & $50^{\circ} 40^{\prime} 27.04^{\prime \prime} \mathrm{W}$ & $\mathrm{X}$ & & \\
\hline & $26^{\circ} 37^{\prime} 4.18^{\prime \prime} \mathrm{S}$ & $50^{\circ} 40^{\prime} 17.00^{\prime \prime} \mathrm{W}$ & $\mathrm{X}$ & & \\
\hline & $26^{\circ} 37^{\prime} 9.73^{\prime \prime} \mathrm{S}$ & $50^{\circ} 40^{\prime} 33.6^{\prime \prime} \mathrm{W}$ & $\mathrm{X}$ & & \\
\hline & $26^{\circ} 37^{\prime} 9.73^{\prime \prime} \mathrm{S}$ & $50^{\circ} 40^{\prime} 33.6^{\prime \prime} \mathrm{W}$ & $X$ & & \\
\hline & $26^{\circ} 37^{\prime} 8.75^{\prime \prime} \mathrm{S}$ & $50^{\circ} 40^{\prime} 42.59^{\prime \prime} \mathrm{W}$ & $\mathrm{X}$ & & \\
\hline & $26^{\circ} 37^{\prime} 9.91^{\prime \prime} \mathrm{S}$ & $50^{\circ} 40^{\prime} 27.01^{\prime \prime} \mathrm{W}$ & $\mathrm{X}$ & & \\
\hline Vargem Bonita & $27^{\circ} 0{ }^{\prime} 24.01^{\prime \prime} \mathrm{S}$ & $51^{\circ} 44^{\prime} 24^{\prime \prime} \mathrm{W}$ & & \multirow{5}{*}{\multicolumn{2}{|c|}{$\mathrm{X}$}} \\
\hline \multirow{4}{*}{ Videira } & $27^{\circ} 0^{\prime} 7.02^{\prime \prime} \mathrm{S}$ & $51^{\circ} 9^{\prime} 33.119^{\prime \prime} \mathrm{W}$ & $\mathrm{X}$ & & \\
\hline & $27^{\circ} 0^{\prime} 27.84^{\prime \prime} \mathrm{S}$ & $51^{\circ} 9^{\prime} 2.55^{\prime \prime} \mathrm{W}$ & $\mathrm{X}$ & & \\
\hline & $27^{\circ} 0^{\prime} 7.34^{\prime \prime} \mathrm{S}$ & $51^{\circ} 9^{\prime} 33.04^{\prime \prime} \mathrm{W}$ & $\mathrm{X}$ & & \\
\hline & $27^{\circ} 0^{\prime} 0.54^{\prime \prime} \mathrm{S}$ & $51^{\circ} 7^{\prime} 12.198^{\prime \prime} \mathrm{W}$ & $\mathrm{X}$ & & \\
\hline
\end{tabular}

Based on the morphological data, an identification chart and a morphological description of each species were elaborated. Macroscopic images of the plants were also performed using a Sony MVC-CD500 digital camera and illustrations of the mericarps were made with ink on butter-paper. The characters used were those that could be observed by the naked eye or with the help of a hand magnifying glass.

Samples of dehydrated Malva species marketed as medicinal plants were acquired from commercial establishments and pharmacies in the Southern Region of Brazil, randomly. For this purpose, 60 samples of commercial packages were purchased, in pharmacies $(70 \%)$, natural products $(25 \%)$ and supermarkets $(5 \%)$. Only $85 \%$ of the products marketed had the proper batches. The label descriptions of each sample were observed. In addition to that, macroscopic and microscopic characteristics (for exemple trichomes), of parts of the plant by comparing them with the characteristics described in the specialized literature were made.

\section{Results}

The visits all over the Western Mesoregion of Santa Catarina State could allow observed 180 specimens of Malva distributed into five species: M. nicaeensis Allioni, M. parviflora L., M. pseudolavatera Webb. \& Berthel, M. sylvestris L., and M. verticillata $\mathrm{L}$.

Three species of Malvaceae and one of Geraniaceae were found from 60 samples collected as dehydrated samples labeled under the common name of mallow, malva-branca (white-mallow), or malva-cheirosa (smelled-mallow). In relation to the Malvaceae species, we identified: M. sylvestris, M. parviflora and Sida cordifolia $\mathrm{L}$.

Material from Pelargonium graveolens L'Hér. ex Aiton was found in $1.66 \%$ of the packages, which is a Geraniaceae (Table 2); a medicinal and aromatic species originated in South Africa (Arrigoni-Blank, Almeida, Oliveira \& Blank, 2011). P. graveolens is widely used in the perfume and cosmetic industries (Saxena et al., 2000). 
Table 2. Species identified on the labels of the marketed packages with their contents

\begin{tabular}{|c|c|c|c|c|}
\hline \multirow{3}{*}{ Botanical name according to the label } & \multicolumn{4}{|c|}{ Species confirmed on the package contentes } \\
\hline & \multicolumn{4}{|c|}{ Numbers in percentage } \\
\hline & Sida cordifolia & Pelargonium graveolens & Malva sylvestris & Malva parviflora \\
\hline M. sylvestris & 40.00 & 0.00 & 15.00 & 1.66 \\
\hline M. crispa* & 0.00 & 1.66 & 0.00 & 0.00 \\
\hline Sida cordifolia & 15.00 & 0.00 & 0.00 & 0.00 \\
\hline Absent & 25.00 & 0.00 & 1.66 & 0.00 \\
\hline Total $(\%)$ & 80.00 & 1.66 & 16.66 & 1.66 \\
\hline
\end{tabular}

Note. * Current name: $M$. verticillata.

\subsection{Key for Identification and Description of Species}

3.1.1 Key for the Identification of Pelargonium graveolens, Sida cordifolia and Malva Species From the Western Mesoregion of Santa Catarina

1-Leaves opposite; adaxial surface trichomes simple Pelargonium graveolens

1-Leaves alternate; adaxial surface trichome fasciculate and simple 2

2-Flowers without involucel; petals not emarginate Sida cordifolia

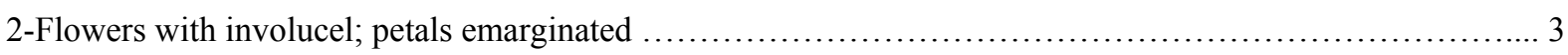

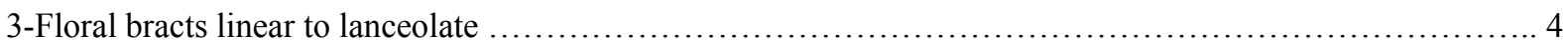

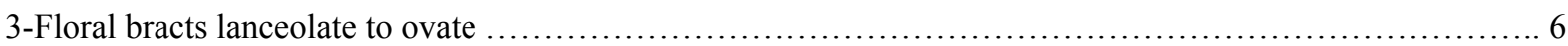

4-Lamina adaxial surface pubescent, trichome fasciculate and simple; mericarps almost

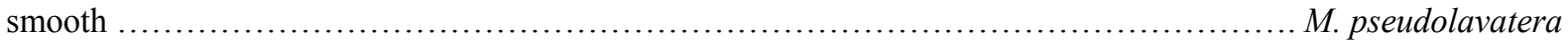

4-Lamina adaxial surface glabrescent, trichome simple; mericarps laterally reticulated ........................ 5

5-Mericarps extremely reticulate, with slight winged projections ............................... Marviflora

5 -Mericarps slight reticulate ............................................................ verticillata

6-Herbaceous erect; petals 7-20 mm long, pinkish; mericarps gently reticulated .................... M. sylvestris

6-Herbaceous frequently decumbent. Petals 6-10 $\mathrm{mm}$ long, purplish to lavender; mericarps slight reticulated M. nicaeensis

\subsubsection{Description of Malva Species Cultivated in the Western Mesoregion of Santa Catarina}

Malva nicaeensis Allioni, Fl. Pedem. 2:40. 1785.

Herbaceous decumbent, rarely erect, 0.7-2.1 m tall. Stipule 2-8 $\times 3-6 \mathrm{~mm}$. Leaves with petioles 4-20 cm long. Lamina 3.3-12 $\times 4-18 \mathrm{~cm}$, reniform to orbicular, rarely lobate, base slight auricle, margin crenate; glabrescent. Flowers 3-9 in the leaf axils. Bractlets of the involucel lanceolate to ovate, ca. $2 \mathrm{~mm}$ wide; calyx 4-7 $\mathrm{mm}$ long, spreading in fruit; corolla purplish to lavender, ca. $8 \mathrm{~mm}$ diameter; petals $0.6-10 \mathrm{~cm}$ long, emarginated. Schizocarp brown when mature, ca. $0.7 \mathrm{~cm}$ diameter, 9-12 mericarps, slight reticulated (Figures 2a and 3a).

Malva parviflora L., Demonstr. pl. 18. 1753.

Herbaceous decumbent to erect, 0.4-1.8 m tall. Stipule 2-8 $\times$ 3-6 mm. Leaves with petioles 3.8-24.5 cm long. Lamina 2.5-12 x 3.3-18 cm, reniform, lobate, base auriculate, margin crenate; glabrescent. Flowers 2-5 in the leaf axils. Bractlets of the involucel linear to lanceolate, ca. $3 \times 2 \mathrm{~mm}$; calyx 2-7 mm long, spreading in fruit; corolla whitish with red center lavender, ca. $0.7 \mathrm{~cm}$ diameter; petals $0.4-0.7 \mathrm{~cm}$ long, emarginated. Schizocarp brown or gray, when mature, ca. $0.7 \mathrm{~cm}$ diameter, 10-11 mericarps, with slight winged projections (Figures $2 \mathrm{~b}$ and 3b).

Malva pseudolavatera Webb. \& Berthel, Hist. Nat. Iles Canaries 3(2(1)):29(-30). 1836.

Herbaceous decumbent to erect, 0.4-2 m tall. Stipule ca. $5 \times 3 \mathrm{~mm}$. Leaves with petioles $4 \times 14.1 \mathrm{~cm}$ long. Leaf blade 3-9.5 $\times 4-17 \mathrm{~cm}$, reniform, lobate, base truncate to slight subcordate, margin crenate; pubescent. Flowers 2-7 in the leaf axils. Bractlets of the involucel linear to lanceolate, ca. $3 \times 2 \mathrm{~mm}$; calyx 5-9 cm long, spreading in fruit; corolla whitish to lavender, maculate, ca. $1 \mathrm{~cm}$ diameter; petals $0.6-2 \mathrm{~cm}$ long, emarginated. Schizocarp brown, ca. $1 \mathrm{~cm}$ diameter, 9 mericarps, almost smooth (Figures $2 \mathrm{c}$ and $3 \mathrm{c}$ ). 
Malva sylvestris L., Sp. Pl. 685. 1753.

Herbaceous erect, $0.5-1.5 \mathrm{~m}$ tall. Stipule ca. $5 \times 3 \mathrm{~mm}$. Leaves with petioles $5-8 \mathrm{~cm}$ long. Lamina 3-9 $\times 3-8 \mathrm{~cm}$, reniform, lobate, base truncate, margin crenate; glabrescent. Flowers 3-8 in the leaf axils. Bractlets of the involucel lanceolate to ovate, ca. $2 \mathrm{~mm}$ wide; calyx 3-6 mm long, slight spreading in fruit; corolla purplish, maculate, ca. $2.5 \mathrm{~cm}$ diam; petals $0.7-2.2 \mathrm{~cm}$ long, emarginated. Schizocarp brown or grey, when mature, ca. 0.7 cm diam., 9-11 mericarps, gently reticulated (Figures $2 \mathrm{~d}$ and $3 \mathrm{~d}$ ).

Malva verticillata L., Sp. P1. 2: 689. 1753.

Herbaceous erect, 1-2.5 m tall. Stipule ca. $5 \times 3 \mathrm{~mm}$. Leaves with petioles 8-19 cm long. Lamina 8-17 $\times 8-18 \mathrm{~cm}$, reniform to lobate, lobate, base auriculate, sometimes with imbricate lobes, margin crenate; glabrescent. Flowers 3-10 in the leaf axils. Bractlets of the involucel linear to lanceolate, ca. $3 \times 2 \mathrm{~mm}$; calyx 3-9 mm long, spreading in fruit; corolla white, maculate in the apex, ca. $0.8 \mathrm{~cm}$ diameter; petals $0.3-0.8 \mathrm{~cm}$ long, emarginated. Schizocarp brown, when mature, ca. $0.8 \mathrm{~cm}$ diam., 10-11 mericarps, slight reticulated (Figures 2e and 3e).

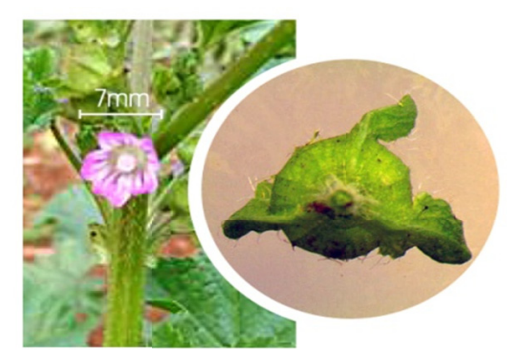

a

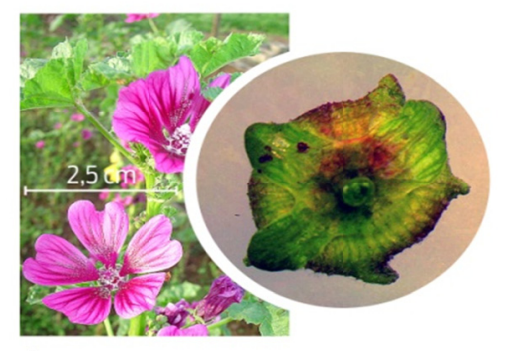

d
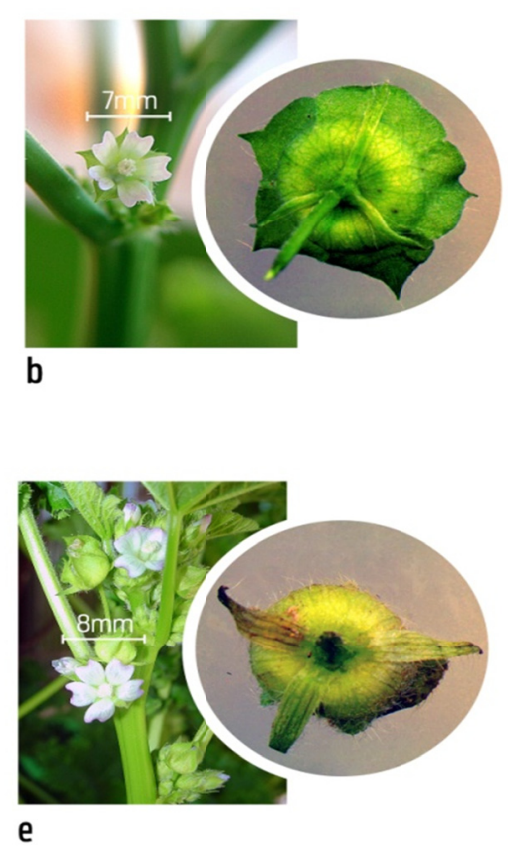

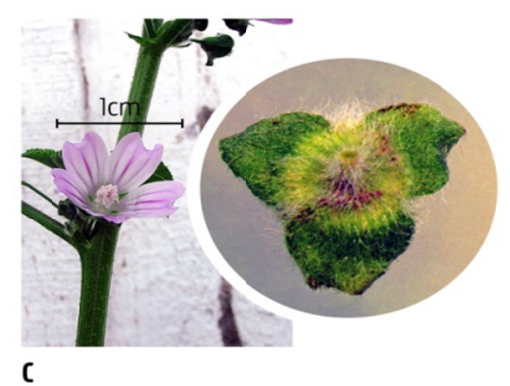

C

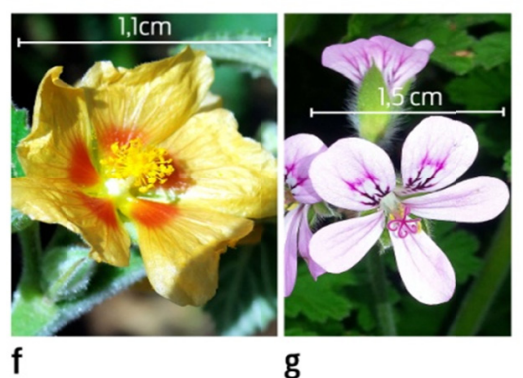

Figure 2. Malva, Sida and Pelargonium flowers

Note. a. M. nicaeensis, flower and detail of the epicalyx; b. M. parviflora, flower and detail of the epicalyx; c. M. pseudolavatera, flower and detail of the epicalyx; d. M. sylvestris, flower and detail of the epicalyx; e. M. verticillata, flower and detail of the epicalyx; f. Sida cordifolia, flower; g. Pelargonium graveolens, flower. 


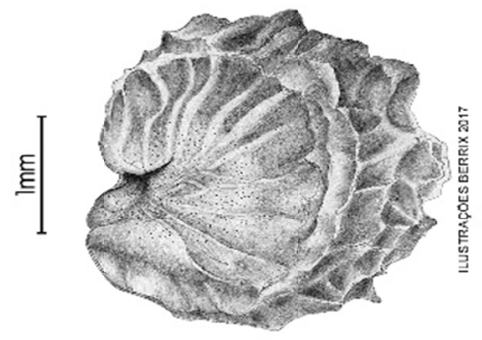

a

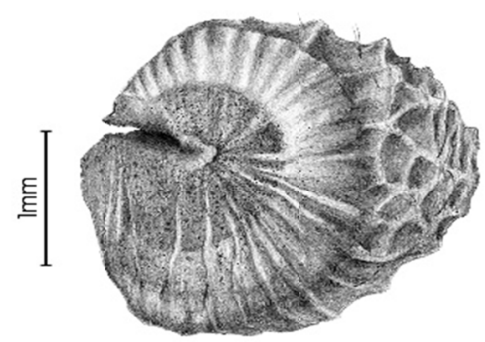

d

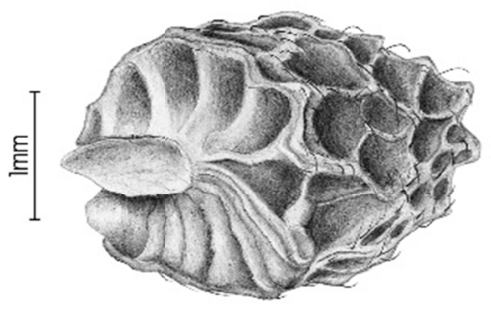

b

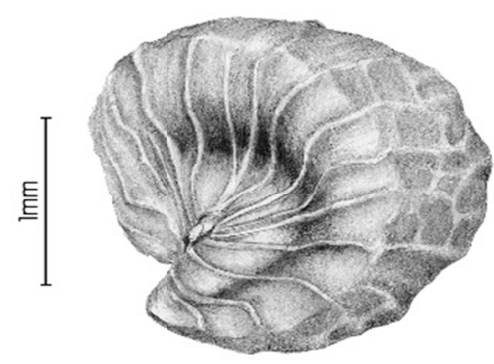

e

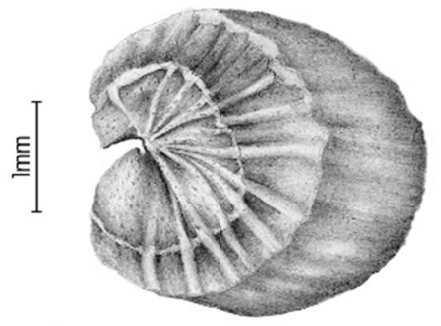

C

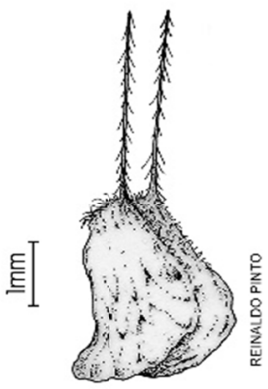

Figure 3. Malva and Sida mericarps

Note. a. M. nicaeensis; b. M. parviflora; c. M. pseudolavatera; d. M. sylvestris; e. M. verticillata; f. Sida cordifolia.

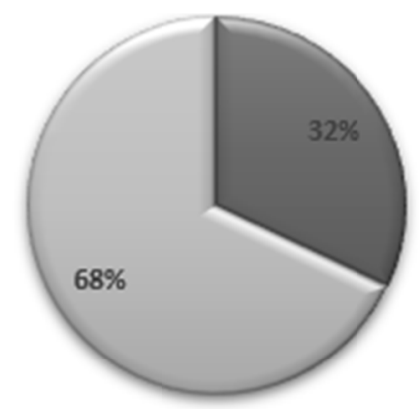

- Species in conformity with package contents

$\square$ Species not in conformity with package contents

Figure 4. Representativeness of the species correctly identified, Souther Brazil, 2015-2017

\section{Discussion}

The most evident morphological difference between M. parviflora and M. sylvestris found in the dried samples was in the mericarp, which varies from slightly reticulate to extremely reticulate with winged projections (Figure 3 ). Whereas other characteristics were not of great relevance. In contrast, S. cordifolia (Figure 2f) is another genus of Malvaceae but very diverse in numbers of species, with 94 species described occurring in Brazil (Flora do Brasil 2020, n.d.). In addition to the yellow flowers with markings, morphological differences between $S$. cordifolia and Malva show that the mericarps in S. cordifolia are long awns with retrorsely trichomes, which is not observed in Malva (Bovini, Carvalho-Okano, \& Vieira, 2001).

P. graveolens, the leaves are opposite zygomorphic flowers and schizocarps with elastic dehiscence, meaning mericarps that are released from the lower portion and are rolled up and get trapped at the apex.

It was found that $15 \%$ of the Malva sylvestris samples labeled under the common name "mallow" ("malva") were correctly identified, and $15 \%$ of those labeled as "malva-branca" and the scientific name of Sida cordifolia were correctly related to the contents of the samples (Table 2). Considering that, M. sylvestris is a widely-cultivated species of mallow and studied regarding its phytochemical aspects. It appears to be the target 
species with the highest non-conformities. S. cordifolia was a species present in $80 \%$ of the samples, with $65 \%$ of them marketed as M. sylvestris or labeled under the common name of "mallow" ("malva").

After this analysis, we can point out a high degree of erroneously identified species at package labels that make up the contents of the analyzed commercial packages. Figure 4 highlights the total number of species correctly identified in relation to the contents of the packages and those that were not correctly identified.

Martins et al. (2015) analyzed samples of M. sylvestris or "mallow" available in dehydrated form in different Brazilian locations by using mass spectrometry and morphological analysis. They identified $50 \%$ of the samples as $S$. cordifolia. The packages labeled as malva-cheirosa and identified as Malva crispa L. (currently, Malva verticillata), have been incorrectly identified. They are, in the fact, Pelargonium graveolens, which are Geraniaceae, but very much resemble M. sylvestris. Romitelli and Martins (2013) and Martins et al. (2015) report that the misuse of $S$. cordifolia can be dangerous to patients with cardiovascular problems, anxiety or behavioral disorders due to the presence of ephedrine, pseudoephedrine and alkaloids.

Colet, Dal Molin, Cavinatto, Baiotto and Oliveira (2015), analyzed 44 packages of medicinal plants commercialized in pharmacies in the municipality of Ijuí/RS, based on the legilations on the subject. Among all the requirements, the results showed that all the samples had common name, while the scientific name in only $75 \%$.

There is a high consuption of $S$. cordifolia and P. graveolens instead of M. sylvestris, which has already been recognized as medicinal due to misunderstandings in the identification of the species. Species of the Malva genus are difficult to recognize after processed. Nevertheless, it is likely better to recognize the species more accurately when based on different parts sampled from the commercial package. The color of the flower, the morphology of the epicalyx's bractole and the morphology of the mericarp are essential for identifying the species.

The high presence of S. cordifolia in the studied samples labeled as other species $(65 \%)$ is probably due to the fact that the species is widely distributed in Brazil throughout all biomes, and occur on the side of paths and roads. Thus, it does not need to be cultivated, which facilitates its collection from several locations in Brazil. Furthermore, it has a slight resemblance to the Malva genus to the untrained eye. On the other hand, the Malva genus occurs under cultivation in environments with lower temperatures and it rarely occurs in the wild ecosystem.

Further studies of this nature are necessary in other states of Brazil in order for the Medicinal Plants Policy to be properly implemented in Brazil.

\section{Conclusion}

Five cultivated Malva species were found in the Western Mesoregion of Santa Catarina State-Southern Brazil: M. nicaeensis, M. parviflora, M. pseudolavatera, M. sylvestris and M. verticillata.

Commercial samples of dehydrated $M$. sylvestris revealed the presence of parts of plants of the family Geraniaceae. S. cordifolia (Malvaceae) is the main mixed species marketed in packages of medicinal plant $M$. sylvestris.

\section{Acknowledgements}

To FAPESC through the Rede Guarani Serra Geral/TO 2015TR1067 project, to Programa UNIEDU de Pós-graduação for the scholarship granted to the first author, to Conselho Nacional de Desenvolvimento Científico

e Tecnológico (CNPq), for an award for excellence in research (process number 304081/2015-9 and 307376/2017-6), to the geologist Ernani Bellon and the illustrator Luiz Berri, both from the Instituto de Pesquisas Jardim Botânico do Rio de Janeiro for the preparation of the map and the illustrations, respectively.

\section{References}

Alves, P. M., Queiroz, L. M. G., Pereira, J. V., \& Pereira, M. S. V. (2009). Atividade antimicrobiana, antiaderente e antifúngica in vitro de plantas medicinais brasileiras sobre microrganismos do biofilme dental e cepas do gênero Candida. Revista da Sociedade Brasileira de Medicina Tropical, 42(2), 222-224. https://doi.org/10.1590/S0037-86822009000200028

Arrigoni-Blank, M. F., Almeida, S. A., Oliveira, A. C. L., \& Blank, A. F. (2011). Micropropagação e aclimatação de gerânio (Pelargonium graveolens L.). Revista Brasileira de Plantas Medicinais, 13(3), 271-275. https://doi.org/10.1590/S1516-05722011000300004 
Baroni, E. (1984). Guida Botanica D'Italia: Chiavi analitiche per determinare le piante spontanee che crescono nella penisola (8th ed.). Bologna, BO: Licinto Cappeli Editore.

Battisti, C., Garlet, T. M. B., Essi, L, Horbach, R. K., Andrade, A., \& Badke, M. R. (2013). Plantas medicinais utilizadas no município de Palmeira das Missões, RS, Brasil. Revista Brasileira de Biociências, 11(3), 338-348.

Benson, B., Rosalen, P. L., Alencar, S. M., \& Murata, R. M. (2015). Malva sylvestris Inhibits Inflammatory Response in Oral Human Cells. An In Vitro Infection Model. PLoS ONE, 10(10). e0140331. https://doi.org/10.1371/journal.pone.0140331

Bovini, M. G., Carvalho-Okano, R. M., \& Vieira, M. F. (2001). Malvaceae A. Juss. no Parque Estadual do Rio Doce, Minas Gerais, Brasil. Rodriguésia, 52(81), 17-47. https://doi.org/10.1590/2175-78602001528102

Brasil, Ministério da Saúde, Agência Nacional de Vigilância Sanitária. (2010). Dispõe sobre a notificação de drogas vegetais junto à Agência Nacional de Vigilância Sanitária (ANVISA) e dá outras providências. Resolução RDC n.10, de 9 de Março de 2010. Diário Oficial da União, 1, 52-59.

Brasil, Ministério da Saúde, Secretaria de Ciência, Tecnologia e Insumos Estratégicos, Departamento de Assistência Farmacêutica. (2006). Política Nacional de Plantas Medicinais e Fitoterápicos. Brasília: Ministério da Saúde. Retrieved from http://bvsms.saude.gov.br/bvs/publicacoes/politica_nacional_ fitoterapicos.pdf

Colet, C. F., Dal Molin, G. T., Cavinatto, A. W., Baiotto, C. S., \& Oliveira, K. R. (2015). Análises das embalagens de plantas medicinais comercializadas em farmácias e drogarias do município de Ijuí/RS. Revista Brasileira de Plantas Medicinais, 17(2), 331-339. https://doi.org/10.1590/1983-084X/13_027

Ecker, A. C. L., Martinsa, I. S., Kirscha, L., Lima, L. O., Stefenonb, L., \& Mozzinib, C. B. (2015). Efeitos benéficos e maléficos da Malva sylvestris. Journal of Oral Investigations, 4(1), 39-43. https://doi.org/ 10.18256/2238-510X/j.oralinvestigations.v4n1p39-43

Escobar, G. P., Schönswetter, P., Fuertes Aguilar, J., Neto Feliner, G., \& Schneeweiss, G. M. (2009). Five molecular markers reveal extensive morphological homoplasy and reticulate evolution in the Malva aliance (Malvaceae). Molecular Phylogenetics Evolution, 50(2), 226-239. https://doi.org/10.1016/j.ympev.2008. 10.015

Farmacopeia Brasileira. (2002). Malva. Farmacopeia Brasileira (Part II) (4th ed.). São Paulo, SP: Atheneu.

Flora do Brasil 2020. (n.d.). Jardim Botânico do Rio de Janeiro. Retrieved from http://floradobrasil.jbrj.gov.br/ reflora/floradobrasil/FB156

Flora-On. (2017). Flora de Portugal Interactiva. Sociedade Portuguesa de Botânica. Retrieved from $\mathrm{http} / / \mathrm{www}$.flora-on.pt

Fryxell, P. A. (1988). Malvaceae of Mexico. Systematic Botany Monograph, 25, 1-522. https://doi.org/10.2307/ 25027717

Hinsley, S. R. (2014). The Malva (Mallow) Pages: Contents and overview. Retrieved from http://www.malvaceae.info/Genera/Malva/Malva.html

Martins, C. A. F., Piantavini, M. S., Ribeiro, R. P., Anano, E., Prá, B. V. D., \& Pontarolo, R. (2015). Non-Targeted Electrospray Mass Spectrometry-Based Metabolic Fingerprinting and PLS-DA-Based Classification of Brazilian "Malvas". Journal Brazilian Chemical Society, 26(2), 365-372. https://doi.org/ 10.5935/0103-5053.20140288

Ray, M. F. (1995). Systematics of Lavatera and Malva (Malvaceae, Malveae) a new perspective. Plant Systematics and Evolution, 198(1), 29-53. https://doi.org/10.1007/BF00985106

Robyns, A. (1965). Malvaceae. Flora of Panama. Annals Missouri Botanical Garden, 52(3), 497-578. https://doi.org/10.2307/2394804

Romitelli, I., \& Martins, M. B. G. (2013). Comparison of leaf morphology and anatomy among Malva sylvestris ("gerânio-aromático"), Pelargonium graveolens ("falsa-malva") and Pelargonium odoratissimum ("gerânio-de-cheiro"). Revista Brasileira de Plantas Medicinais, 15(1), 91-97. https://doi.org/10.1590/ S1516-05722013000100013 
Saxena, G., Banerjee, S., Rahman L., Mallavarapu, G. R., Sharma, S., \& Kumar S. (2000). An efficient in vitro produce for micropropagation and generation of somaclones of rose scented Pelargonium. Plant Science, 155(2), 133-140. https://doi.org/10.1016/S0168-9452(00)00213-2

The Plant List. (2013). Malvaceae (Version 1.1). Retrieved from http://www.theplantlist.org/tpl1.1/search? $\mathrm{q}=$ malva

Thiers, B. (2017). Index Herbariorum: A global directory of public herbaria and associated staff. New York Botanical Garden's Virtual Herbarium. Retrieved from http://sweetgum.nybg.org/science/ih

\section{Copyrights}

Copyright for this article is retained by the author(s), with first publication rights granted to the journal.

This is an open-access article distributed under the terms and conditions of the Creative Commons Attribution license (http://creativecommons.org/licenses/by/4.0/). 